\title{
DNA SYNTHESIS IN RYE CHROMOSOMES
}

\author{
U. AYONOADU* and $H$. REES \\ Deportment of Agricultural Botany, University College of Woles, Aberystwyth
}

Received 19.v.72

\begin{abstract}
SUMmary
Following the uptake of tritiated thymidine during the latter part of the synthesis $(S)$ phase of mitosis the distribution of label at metaphase varies between chromosomes and between segments within chromosomes. Per unit volume $\mathrm{B}$ chromosomes are more densely labelled than the normal, A chromosomes of the rye complement. Within A chromosomes the terminal segments and centromere regions are the most heavily labelled.

Analyses are presented which distinguish between the different causes of heavy labelling. In terminal segments of the long arms late completion of DNA synthesis in heterochromatin contributes to the heavy labelling. In contrast the disproportionately heavy labelling in $B$ 's is attributable to a higher DNA density in $B$, as compared with A chromosomes, at metaphase. It is probable that the heavy labelling around the centromeres of rye chromosomes is, also, a reflection of a higher DNA density at metaphase. Our evidence indicates it is not due to a late completion of DNA synthesis.

B chromosomes have no influence upon the pattern of labelling in A chromosomes. It is possible that they reduce the rate of DNA synthesis during $S$.
\end{abstract}

\section{INTRODUCTION}

LABELLING with tritiated thymidine has proved a useful device for determining the course of DNA synthesis during interphase within chromosome complements. Each species has its own distinctive pattern. In some the evidence indicates that synthesis begins at the ends of the chromosomes and proceeds inwards towards the centromeres, e.g. Crepis capillaris (Taylor, 1958); in Tradescantia paludosa the reverse (Wimber, 1961). Other species show an asynchronous replication pattern of greater complexity, with much variation between individual chromosomes (e.g. in man, Lima-de-Faria et al., 1961). Particularly marked is the asynchronous synthesis of DNA in heterochromatin as compared with euchromatin. In the former the DNA is synthesised later, a fact first reported by Lima-de-Faria (1959) in Melanoplus and rye.

The pattern of asynchrony in DNA synthesis is established from the distribution of tritium label at metaphase. There are, however, causes other than asynchrony which affect the distribution of metaphase label. We present analyses which distinguish between the different causes. The analyses are used to describe and compare the pattern of DNA replication at late $S$ in two kinds of rye, namely plants with and without B chromosomes.

\section{Materials AND Methods}

Rye seeds containing $0 \mathrm{~B}$ and $2 \mathrm{~B}$ chromosomes were germinated in petri dishes at room temperature. The seedlings were immersed in tritiated

* Present address: Bunda College of Agriculture, University of Malawi, Lilongwe, Malawi. 
thymidine $(2 \mu \mathrm{c} . / \mathrm{ml}$.) for 30 minutes, washed thoroughly in distilled water and pre-treated with 0.1 per cent. colchicine for 6 hours. At $20^{\circ} \mathrm{G}$. the duration of $G 2$ (the post synthesis phase) in rye is 3 to 4 hours. The duration of synthesis $(S)$ is 5 to 6 hours (see Ayonoadu and Rees, 1968). This means that the chromosomes scored were being labelled during the latter part of $S$ only. The roots were fixed in $1: 3$ acetic alcohol, hydrolysed for 12 minutes in $1 \mathrm{~N} \mathrm{HCl}$ at $60^{\circ} \mathrm{C}$., and stained in basic fuschin for 1 hour. Root tip squashes were made on previously subbed slides and the coverslips removed on dry ice. Stripping film (Kodak AR 10) was applied and the slides stored in the dark at $4^{\circ} \mathrm{C}$, for 3 weeks. Autoradiographs were developed (Kodak D198 developer) for 6 minutes and fixed for 12 minutes.

For the purpose of mapping the distribution of silver grains, each chromosome of the normal, $A$ complement was divided into seven segments, a centromere segment $(C)$ and, in each arm, three segments of equal length (fig. 1). Data for all chromosomes within cells were pooled because classifi-

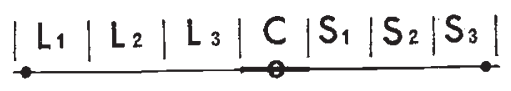

Fig. 1.-Arbitrary subdivision of the rye chromosome into seven segments. Segments in the short arm $(S 1, S 2$ and $S 3)$ are of course shorter than long-arm segments.

cation of the rye complement at metaphase is uncertain. Two experiments (I and II) involved plants without B chromosomes, the other (III) plants with two B chromosomes. Grain counts over B's are presented separately.

\section{Results}

The distributions of silver grains over the seven A chromosome segments are presented in fig. 2. There is a considerable variation between segments. The end segments, $L 1$ and $S 3$, and the centromere segment $(C)$ are heavily labelled relative to the others. $\chi^{2}$ analyses confirm that the variation between segments is significant ( $\mathrm{P}=<0.001$ in each experiment).

The labelling pattern agrees closely with the results of Darlington and Haque (1966) and Lima-de-Faria and Jaworska (1972). The heavy labelling in terminal segments is no doubt associated with the prominent heterochromatic telomeres in rye chromosomes (Lima-de-Faria, 1959; Darlington and Haque, 1966). There is controversy as to whether the centromere region, also heavily labelled, is strictly heterochromatic (Lima-de-Faria, 1959) or merely precocious in coiling during prophase (Darlington and Haque, 1966) and thereby denser in DNA content at metaphase. We shall return to this subject in a later section.

\section{(i) Variation due to $B$ chromosomes}

It will be seen that the pattern of labelling is consistent for all three experiments (fig. 2). The consistency is confirmed by a $\chi^{2}$ analysis which shows no significant heterogeneity $(P=0 \cdot 3-0 \cdot 5)$. We conclude that $\mathrm{B}$ chromosomes have no influence on the distribution of label and, hence, upon the pattern of DNA synthesis within A chromosomes. We note, at the 
same time, that the amount of label is much less in experiment III than I or II. It could be argued that the rate of DNA synthesis is reduced in the presence of B's. This would be compatible with the fact that the duration of the mitotic cycle (including $S$ ) is extended in the presence of $\mathrm{B}$ chromosomes (Ayonoadu and Rees, 1968). It would, however, be wrong to put too much

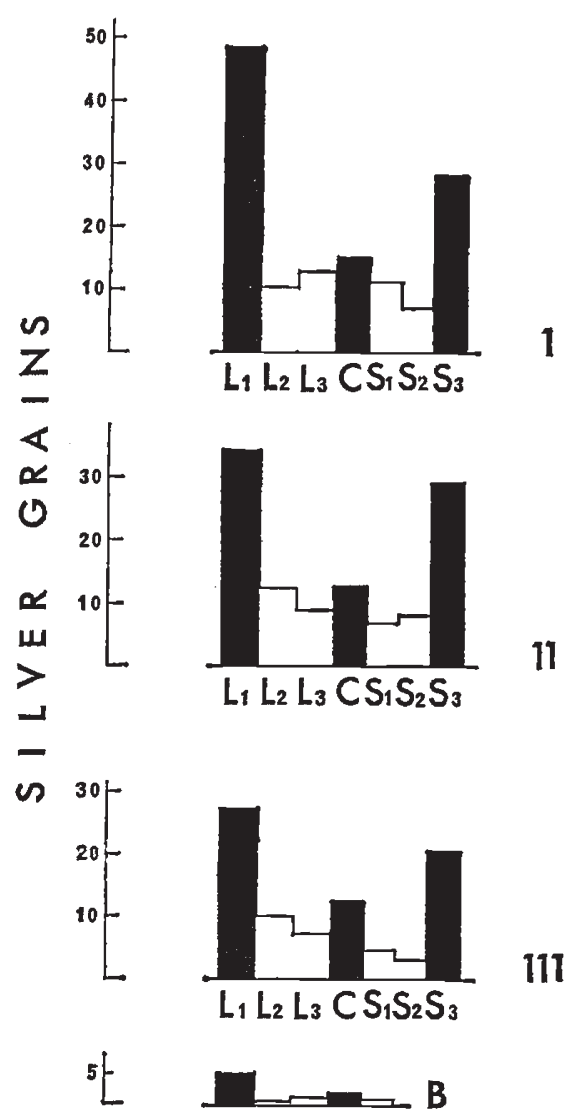

FIG. 2.-The distribution of silver grains over rye chromosomes at metaphase following the incorporation of tritiated thymidine during the latter part of $S$ (the synthesis phase). The mean silver grain counts per segment are derived from data pooled for chromosomes within cells. In I and II are plants without B chromosomes. In III the data are from plants with two B chromosomes. The distribution of silver grains over B chromosomes is given separately.

weight on this interpretation because the experiments, although comparable in terms of procedure, were carried out at different times. Consequently any effects due to B's would be confounded and inseparable from any effects resulting from uncontrollable variables in experimental conditions.

Turning to the labelling pattern within B's themselves (fig. 2), it agrees very closely with that described by Darlington and Haque, 1966). The heavy labelling in the terminal segment of the long arm corresponds with the location of heterochromatin in that arm. 


\section{(ii) Variation in labelling; cause and effect}

It is often asserted that chromosomes or segments of chromosomes which are relatively heavily labelled at metaphase following the uptake of tritiated thymidine during late $S$ are those completing synthesis of their DNA later than the less heavily or lightly labelled component of the chromosome complement. This evidence does not in itself, however, justify the assertion, as Barlow and Vosa (1969) in particular have emphasised. Altogether there are three possible causes of the disproportionately heavy labelling. They are:

1. Late completion of DNA synthesis, as already mentioned.

2. A higher rate of DNA synthesis (but without extension of the DNA synthesis period).

3. A greater DNA density at metaphase in the heavily labelled regions.

To distinguish between the three causes is difficult. A useful approach to the problem has been put forward by Barlow and Vosa (1969). Their method makes it possible to determine whether or not late completion of DNA synthesis accounts for the heavy labelling. It is based on the following reasoning. First, the number of silver grains per chromosome or chromosome segment at metaphase will be reduced progressively as the cells which incorporated the tritiated thymidine proceed towards the completion of $S$. If we plot the number of grains in one particular segment or chromosome against the number of grains in another, for each metaphase, the regression line will pass through the origin if synthesis is completed simultaneously in the two segments or chromosomes. Conversely, the regression line will deviate from the origin where synthesis is prolonged in one relative to the other. We have applied this method to our present results.

\section{A chromosomes}

In figs. $3 a, b$ and $c$ we have plotted the grain distributions in $L 1, C$ and $S 3$ (the most heavily labelled segments) against, in each instance, the grain distributions in $S 2$, the segment with least label. There is a significant curvilinearity in respect of $S 3$, the mean square for the quadratic item in the regression analysis being significant at the 1 per cent. level.

When we test for deviation of the intercepts of the regressions from the origin we find significance only for the regression of $L 1$ on $S 2(\mathrm{P}=<0.01)$. Late completion of DNA synthesis is clearly the cause of the heavy labelling in this segment although not necessarily the exclusive cause. Turning to $C$ and $S 3$ there is, in neither case, a significant departure from the origin. Late completion of synthesis cannot, in either case, therefore account for the heavy labelling at late $S$. We must invoke one of the other two alternative causes, namely a higher rate of DNA synthesis or else a greater DNA density within these segments at metaphase.

We cannot compare rates of synthesis in different chromosome segments unless we have knowledge of the variation in density. The latter, in rye, is likely to be considerable. In the centromere region, for example, there is undoubtedly a precocity in coiling during prophase which is very likely to achieve a denser "packing" of DNA in that region by the time the chromosomes reach metaphase. Indeed the findings of Heneen and Casperson quoted by Lima-de-Faria and Jaworska (1972) show, conclusively, well 
defined DNA density gradients along the rye chromosomes at prophase of mitosis. The work on human chromosomes shows that such density gradients persist, to a pronounced degree, to metaphase (see Du Praw, 1970). The consequencies of a varying DNA density at metaphase are easily envisaged.
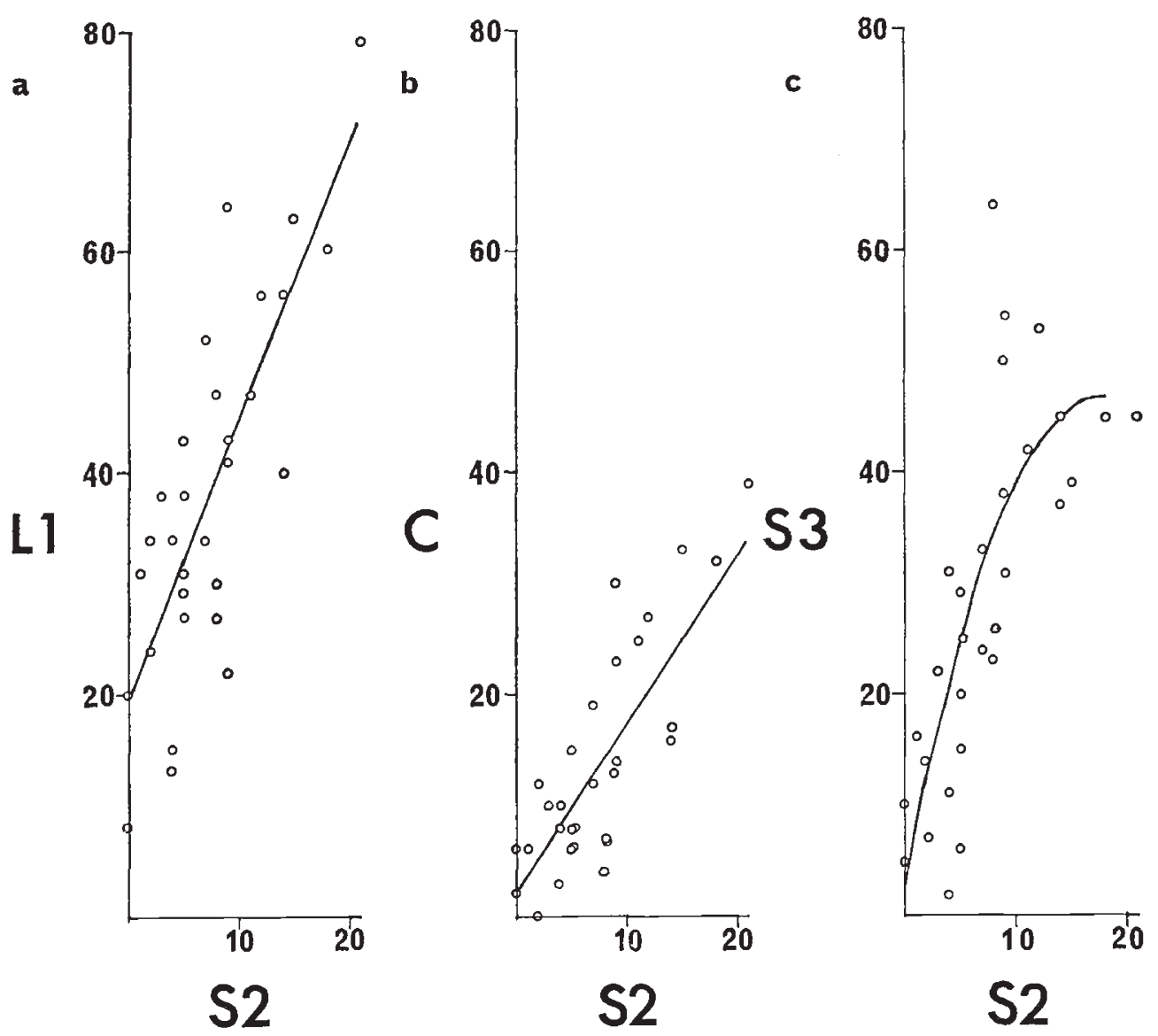

FIG. 3.-Grain numbers in the heavily labelled segments, $L 1, C$ and $S 3$, splotted against the number of grains in the lightly labelled segment, $S 2$. Data pooled from 15 metaphases from both experiments I and II.

They are illustrated in fig. 4. In the diagram it is assumed that DNA replication is synchronous between chromosomes and between segments within chromosomes. It will be observed that the distribution of grains at metaphase will now directly reflect the tightness of coil, the density of DNA. Where the DNA density varies between chromosomes and between segments of chromosomes so also does the intensity of labelling. From the diagrams it is manifestly obvious that a sharp discontinuity in labelling pattern may arise quite independently of asynchrony in DNA replication within a chromosome complement.

We have not, unfortunately, information on the variation in DNA density between segments within rye chromosomes at metaphase. We have, however, information on the DNA density variation between $B$ chromosomes at 
metaphase relative to that of the normal A chromosome complement. The information allows us to test and establish directly the consequence of varying DNA density upon the labelling pattern at metaphase.

$B$ chromosomes versus $A$ 's

Table 1 shows, per unit volume, 1.4 times $\left(6.8\right.$ to 4.8 per $\left.\mu^{3}\right)$ as many silver grains over $\mathrm{B}$ chromosomes than $\mathrm{A}$ chromosomes. In fig. 5 we have

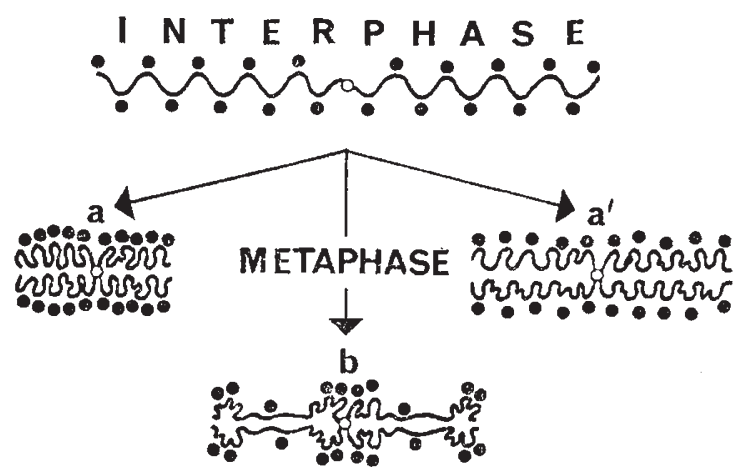

Fig. 4.-The consequences of variation in coiling between chromosomes ( $a$ and $a^{\prime}$ ) and segments of chromosomes $(b)$ upon the intensity of labelling following the incorporation of tritiated thymidine during $S$.

plotted the number of silver grains over B's against the number over A's in each of the 12 metaphases scored. The regression line passes very near to, and does not deviate significantly from, the origin $(P=0 \cdot 2-0 \cdot 1)$. We conclude that DNA synthesis within B's is completed more or less simultaneously with that in A's. The high intensity of label in B's is not, therefore, attributable to late completion of DNA synthesis.

\section{TABLE 1}

The total number of silver grains over the $A$ chromosomes and over two $B$ chromosomes in 12 metaphases in experiment III. Also, the volumes of $A$ and $B$ chromosomes and mean number of silver grains per unit volume

Silver grains
Volume at metaphase
Grains per $\mu^{3}$

$\begin{array}{cc}\text { A chromosomes } & \text { B chromosomes } \\ 1052 & 109 \\ 217 \mu^{3} & 16 \mu^{3} \\ 4.8 & 6 \cdot 8\end{array}$

The DNA densities at metaphase for A and B chromosomes have been calculated by Jones and Rees (1968). In relative terms B's contain 1.5 times as much DNA per unit volume as A's, a figure which corresponds very closely indeed to the ratio of grain counts per unit volume for B's as compared with A's. On the basis of grain counts per unit DNA (as distinct from unit volume) the values for A's and B's are very similar indeed. The disproportionately heavy labelling in $\mathrm{B}$ chromosomes is therefore more apparent than real. It 
is mainly, if not entirely, attributable to their greater DNA density at metaphase.

To turn, in passing, to an unrelated point we have earlier reported that the presence of $\mathrm{B}$ chromosomes in rye brings about an increase in the duration of the mitotic cycle in root tip meristems, an increase over and above that expected simply on the grounds of quantitative increase in nuclear DNA due to B's (Ayonoadu and Rees, 1968). One possible explanation put forward was that B's completed synthesis later than A chromosomes and thereby extended the $S$ phase and, in consequence, the duration of the total cycle. In view of the findings above we must conclude that the control imposed by $\mathrm{B}$ chromosomes is not of this kind. Instead we must conclude that the control is, in part at least genotypic, reflecting the influence of B chromosome "genes" upon the replication of DNA within the complement as a whole.

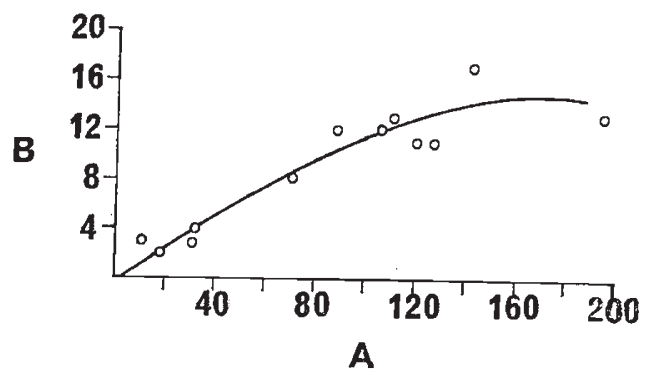

Fig. 5.-The number of silver grains over two B chromosomes plotted against the number of silver grains over the A chromosome complement in each of 12 metaphases (experiment III).

\section{Discussion}

We have been at pains to stress the importance of taking account of factors other than asynchrony of DNA replication in interpreting the variation in labelling pattern within chromosome complements following the uptake of tritiated thymidine during interphase. We have shown that differences in coiling during prophase and thereby in DNA density at metaphase contribute substantially to the variation in labelling intensity between chromosomes (B's versus A's). There is every reason to suppose that density gradients within chromosomes at metaphase also contribute to the variation in labelling intensity.

In their very recent paper Lima-de-Faria and Jaworska (1972) come to the conclusion that regions of chromosomes comprising large chromomeres (the telomeres and centromere regions) begin replicating their DNA later than others and continue to replicate their DNA when other regions have stopped. There is no questioning their conclusion of late commencement of DNA synthesis in these segments. It is based on a detailed and thorough comparison of labelling patterns in metaphase of chromosomes labelled at various intervals during the $S$ phase of the preceding interphase. Their evidence for late completion of DNA synthesis is less convincing. In order to establish that segments or chromosomes continue synthesising DNA when others have ceased requires more than a demonstration of heavy labelling in these segments. The approach of Barlow and Vosa (1969), which we have 
described, coupled with analyses of variance of the regressions involved fulfils the purpose admirably. It will be recalled that when we applied this method to our rye results we found confirmation of late completion of DNA synthesis in the telomeres of long arms as reported by Lima-de-Faria and Jaworska (1972). Elsewhere in the complement, however, we found no evidence for prolonged DNA synthesis. The heavy labelling in some chromosome regions we attributed to a high DNA density at metaphase rather than to late synthesis. We would argue that, in the present context, the significance of the large size of chromomeres associated with these regions lies as testimony to their greater density, the inevitable consequence of which is correspondingly dense labelling following the uptake of tritiated thymidine at late $S$. It does not necessarily follow that they are late in completing their DNA synthesis.

Acknowledgment.-We are indebted to Mr D. A. East for help and advice on statistical procedures.

\section{REFERENCES}

AYONOADU, U., AND REES, H. 1968. The regulation of mitosis by $\mathrm{B}$ chromosome in rye. Exp. Cell. Res., 52, 284-290.

BARLOW, P. H., AND VOSA, G. G. 1969. The pattern of DNA replication in the chromosomes of Puschkinia libanotica. Chromosoma, 28, 457-467.

DARLington, C. D., AND HAQUE, A. 1966. Organisation of DNA synthesis in rye chromosomes. Chromosomes Today, 1, 102-107.

DU PRAW, E. J. 1970. DNA and Chromosomes. Holt, Rhinehart and Wiston, Inc., New York.

JONES, R. N., AND REES, H. 1968. The influence of B-chromosomes upon the nuclear phenotype in rye. Chromosoma, 24, 158-176.

LIMA-DE-FARIA, A. 1959. Differential uptake of tritiated thymidine into hetero- and euchromatin in Melanoplus and Secale. 7. Biophys. Biochem. Cytol., 6, 457-466.

LIMA-DE-FARIA, A., REITALU, J., AND BERGMAN, s. 1961. The pattern of DNA synthesis in the chromosomes of man. Hereditas, 47, 695-704.

LIMA-DE-FARIA, A., AND HALINA JAWORSKA. 1972. The relation between chromosome size gradient and the sequence of DNA replication in rye. Hereditas, 70, 39-58.

TAYLOR, J. H. 1958. The mode of chromosomal duplication in Crepis capillaris. Exp. Cell. Res., 15, 350-357.

WIMBER, D. E. 1961. Asynchronous replication of deoxyribonucleic acid in root tip chromosomes of Tradescantia paludosa. Exp. Cell. Res., 23, 402-407. 